\title{
A Comparison of Soybean Oil Methyl Ester and Diesel Sprays Behavior and Atomization Characteristics \\ Noor Mohsin Jasim
}

\author{
Mechanical Engineering Department, College of Engineering, Kufa University \\ Abstract
}

The present numerical study compares between spray characteristics of diesel and soybean oil methyl ester (SME biodiesel) under non-evaporating sprays. The spray structure of diesel and biodiesel fuel (soybean oil) in a common rail injection system are investigated and compared with that of available experimental data used image processing and atomization performance analysis. The proposed approach for the liquid phase, which based on the sprays' statistical properties, is used to present the gas and liquid phases in an Eulerian-Eulerian approach. The main concept for this model is the possibility of describing a poly disperses spray by using moments of a drop number size distribution function. The main reason for less spray tip penetration in the (SME) comparing with diesel because a larger droplet diameters is the higher density, surface tension and viscosity of (SME). The fuel properties effect on the near nozzle structure is studied. The comparisons are referring that the spray drag, breakup and collision processes are promoted.

Keywords: Spray modeling, biodiesel spray, spray structure, Drop number size. 


\section{Introduction}

Environmental interest and energy conservation have become crucial issues in industrial and automotive fields. Therefore alternative fuel resources are being passively researched as part of an effort to reduce the side effects of emissions and environmental problems. The common understanding of the necessity to use a clean, biodegradable and renewable fuel as alternative fuel has led to present the biodiesel as outstanding solution for the energy security and environment problems. During the last two decades, a big effort has been spent on the effect of alternative fuel properties. In the same way, biodiesel can improve the thermal efficiency through the optimum combustion process and reduced exhaust emission characteristics of diesel engine, which is effected by fuel properties, and spray atomization characteristics. Applicative research based on using different biofuels expressed that the fuel with higher viscosity, density and surface tension produces large cone angle and shorter spray tip penetration [1], [2], [3],[4] and $[5]$.

Experimental and numerical work have been carried out by Park et al. [6] to study the spray and atomization characteristics of an undiluted biodiesel fuel. They used a visualization system to analyses spray area, spray tip penetration and centroid variations while the droplet distribution and droplet size was measured using a droplet measuring system. The KIVA-3V code was used to implement the numerical part. They found that an increase in the injection

pressure leads to decrease in the injection delay because the injection pressure increases the spray fuel.

Majhool and ALJeebori .[7] studied numerically the modelling of spray of biodiesel in terms of spray moment framework. Their investigations were carried out for biodiesel spray under transient engine conditions. The spray tip penetration of biodiesel was compared with diesel fuel in a diesel engine under transient conditions. A comparison was made for highly ambient pressure case between the predicted results and experimental data. The EulerianEulerian approach was used in this study for the simulation of two-phase flow. One parameter was used to analyse and to study the characteristics of the spray through the test process and tracking the behaviour of the biodiesel. 


\section{Method}

\subsection{Moments theory}

Beck and Watkins [8] presented their approach based on the droplet number size distribution; $\mathrm{n}(\mathrm{r})$ represents the multiple of the droplet number probability distribution of droplet radius as shown below:

$$
n(r)=\int_{r_{\min }}^{r_{\max }} N(r) \mathrm{d} r
$$

where $N(r)$ represents the distribution of the droplet number probability. The integral over all droplets gives the total number of droplets per unit total volume (not unit liquid volume). This can be written as below:

$$
Q_{0}=\int_{0}^{\infty} n(r) \mathrm{d} r
$$

This is the first moment of the distribution function. In this approach, the three remaining distribution function moments are defined as below:

$$
Q_{i}=\int_{0}^{\infty} r^{i} n(r) \mathrm{d} r
$$

At a certain point in space and time, $4 \pi \mathrm{Q} 2$, is the total surtace area of the drops , $4 \pi \mathrm{Q} 3 / 3$ is the total volume of the drops, Q0 is the total number of drops, and Q1, is the total sum of

momı $\frac{V_{\text {liquid }}}{V_{\text {liquid }}+V_{\text {gas }}}=\frac{4 \pi}{3} Q_{3}=1-\Theta$ . The fourth

where $\Theta$ is the gas volume fraction. Aditionally, these four parameters gives all mean droplet diameters from D10 to the Sauter mean diameter D32, as, by definition

$$
D_{p q}^{p-q}=\frac{2^{p-q} Q_{p}}{Q_{q}}
$$




\section{No.16 Journal of Petroleum Research \& Studies}

(JPR\&S)

\section{Gamma distribution}

The general gamma number size distribution is given by

$$
n(r)=Q_{0} \frac{(k+2)^{k}}{\Gamma(k) r_{32}} r^{k-1} e^{-(k+2) \frac{r}{r_{32}}}
$$

and

$$
\Gamma(k)=\int_{0}^{\infty} x^{k-1} e^{-x} d x
$$

where $\Gamma(\mathrm{k})$ is the gamma function and $\mathrm{r} 32$ is the Sauter mean radius of the number size distribution of the drops. This is defined by $\mathrm{r} 32=\mathrm{Q} 3 / \mathrm{Q} 2$. For numerical calculations, the gamma function can be approximated by [9]:

$$
\Gamma(k)=\left(\left(\frac{k}{e}\left(k * \sinh \left(\frac{1}{k}+\frac{1}{810 k^{6}}\right)\right)^{1 / 2}\right)^{k}\right)\left(\frac{2 \pi}{k}\right)^{1 / 2}
$$

with an error of at most $1 \%$ for values of $\mathrm{k}>1.0$. With three moments calculated through transport equations, there are two parameters $(\mathrm{Q} 3 / \mathrm{Q} 2=\mathrm{r} 32)$ and $(\mathrm{Q} 2 / \mathrm{Q} 1)$ available to determine r32and k. Insertion of (18) into (2)and partial integration leads to

$$
Q_{i}=\frac{(k+2) Q_{i+1}}{(k+i) r_{32}}
$$

hence, setting $\mathrm{i}=1$,

$$
k=\frac{2 Q_{r}-1}{Q_{r}-1}
$$




\section{No.16 Journal of Petroleum Research \& Studies}

(JPR\&S)

and

$$
Q_{r}=\frac{Q_{2}^{2}}{Q_{1} Q_{3}}
$$

Q0 : is calculated from equation (21), by setting $\mathrm{i}=0$. The gamma distribution is defined for all $\mathrm{k}>0$. However, in practice there are a number of restrictions that must be applied, arising from the sub-models employed; in particular, the drag model break-up and collision models used. The sub-models are derived in details in [10].

\subsection{Transport equations}

The required convection velocity is thus seen to be the average value of the expected moment (denoted by subscript 3), and the equation clearly represents a liquid-phase continuity equation. The source term in the transport equation has only one contribution term due to evaporation. The other phenomena were found to have no effect on the total mass of liquid. A similar form is seen for the remaining moments equations, however, a careful attention has to be given as more source terms created because of the evaporation, droplet breakup effect, changes in the droplet density, and droplet - droplet collisions. The equations can be written as following:

$$
\frac{\partial}{\partial t}\left(Q_{i}\right)+\frac{\partial}{\partial x}\left(Q_{i} U_{l i j}\right)=-S_{Q_{i}}
$$

The using of the $\mathrm{i}$-th moment-average velocity in the equation must be considered. The liquid mass-average velocity, or equation of liquid momentum, as employed in the numerical scheme, is based on the study of Harlow and Amsden [11] for particulate flows. Equation is derived starting from the Lagrangian form of the equation for a group of droplets with identical properties, and the details of derivation of this equation appeared in Beck and Watkins [12]. In brief, the liquid phase momentum equation is written as 


$$
\begin{aligned}
\frac{\partial}{\partial t}\left(\rho_{l}(1-\Theta) U_{l 3 i}\right)+\frac{\partial}{\partial x_{j}} & \left(\rho_{l}(1-\Theta) U_{l 3 i} U_{l 3 j}\right)+U_{l 3 i} S_{m} \\
& =\frac{\partial}{\partial x_{j}}\left(\rho_{l}(1-\Theta) \sigma_{v} \nu_{l}\left(\frac{\partial U_{l 3 i}}{\partial x_{j}}+\frac{\partial U_{l 3 j}}{\partial x_{i}}\right)\right)-S_{U_{i}}
\end{aligned}
$$

Where $\sigma \mathrm{v}$ is the coefficient of Melville and Bray [12], $v \mathrm{l}$ is the turbulent equivalent viscosity; Sm and S Ui are the source terms of mass and momentum, respectively. The remaining equations for the moment-average velocity are derived in a similar manner as described by Beck and Watkins [12].

\section{Test case}

According to the experiments of Kim et al. [13], a fix volume combustion chamber is used to test the non-reactive diesel spray. Droplet velocities and size are measured using Particle Doppler Analyser instrumentation.The injection pressure starts at $50 \mathrm{MPa}$ and varies with needle position in nozzle. The trapped pressure is $0.1 \mathrm{MPa}$ and the nozzle diameter is $0.3 \mathrm{~mm}$. This tests is carried out for different initial gas phase pressures but one case here is adopted as shown in table (1) where the initial gas temperature is $293 \mathrm{~K}$. Table (2) shows adopted biodiesel properties which are given by Kim et al.[13].

Table 1: Physical conditions for the parametric tests and experimental validations (Data of Kim et al. [13].)

\begin{tabular}{ll}
\hline & Kim et al \\
\hline Injection system- & Common- \\
Fuil & Biodiesel \\
Nozzle Type- & Mini-sac \\
Number of nozzel holes- & Single hole \\
Injection Pressure $(M P a)$ & 50.0 \\
Nozzle Radius $(\mathrm{mm})$ & 0.3 \\
Chamber Length $(\mathrm{mm})$ & 100.0 \\
Chamber Radius $(\mathrm{mm})$ & 20.0 \\
Injection SMR $(\mu \mathrm{m})$ & 25.0 \\
Time Step ( $\mathrm{Mec})$ & 2.0 \\
Injection duration $(\mathrm{msec})$ & 1.2 \\
Temperature of $\mathrm{Gas}(\mathrm{K})$ & 293 \\
Gas Pressure $(\mathrm{MPa})$ & 0.10 \\
Gas Density $\left(\mathrm{kg} / \mathrm{m}^{3}\right)$ & 13.8 \\
\hline
\end{tabular}


Table 2: Properties of biodiesel (Data of Kim et al. [13].)

\begin{tabular}{ll}
\hline & Kim 1 \\
\hline Density $\mathrm{kg} / \mathrm{m}^{3}$ & 884 \\
Viscosity $\mathrm{mm}^{2} / \mathrm{sec}$ & $4.0-6.0$ \\
Surface tension $\mathrm{kg} / \mathrm{sec}^{2}$ & 0.028 \\
Boiling point temperature $K$ & $588-623$ \\
Flash point temperature $K$ & $373-443$ \\
Cetane number - & $48-65$ \\
\hline
\end{tabular}

\section{The complete algorithm}

The PISO algorithm of Issa [14] was used as a solution procedure to solve the current problem. This algorithm is coupled with the liquid phase equations. This algorithm is an alternative to SIMPLE and SIMPLEC algorithms in terms of decreasing the large number of total iterations required by these algorithms to solve the gas-phase pressure equation. In this algorithm, the liquid equation is solved only once, at the beginning of the time step. In this approach, the source term caused by drag effect is solved instantaneously at the end of each the time step.

Starting from an initial pressure field, the PISO algorithm consists of the following steps:

- Step 1 . The gas phase velocity fields are calculated from the discretized gas phase momentum equations.

- Step 2. The first set of Pressure Correction Equations (PCE) derived from the discretized continuity equation are solved (see Issa, [14] for details) to obtain the correction gas phase pressures, densities and velocity fields.

- Step 3. The second set of (PCE) is solved to correct the gas phase pressures, densities and velocity fields.

- Step 4. All discretized transport equations are solved (e.g. the turbulence kinetic energy and its dissipation rate).

- Step 5. The transport equations for the moment-average liquid velocities, U11, U12 and U13, are also solved. 
- Step 6. The inter-phase drag, breakup and collision source terms are obtained.

- Step 7. The transport equations for the moments Q1, Q2and Q3of the drop size distribution and for the liquid are solved. The void fraction value is corrected.

\section{Results and discussions}

Before considering the computational results extracting from the in-house code written in FORTRAN-2003. The code is able to read structured or unstructured grid created by GAMBIT with neutral extension. GAMBIT software is used to create a non-uniform grid with a grid refinement strategy. Grid refinement at certain places can be produced based on enlarge grid resolution by increase the compression ratio or number of intervals. Figure (1) shows the computational domain used in this simulation. The grids refinement is applied to capture flow details at a particular space of the interested area. The details of the specified computational domain are: (Volumes 1906), (Faces 3901), (Vertices 1996) and (Injection cells 5).

The diesel and biodiesel spray structure elements which are investigated in this study include spray tip penetration, spray velocity (both components axial and radial) and spray Sauter mean diameter. The validation of the proposed numerical solution is discussed in this section. The simulation results are compared with the experimental available results for the biodiesel Soybean Oil Methyl Ester (SME) have been taken from the literature presented by Kim et al. [13].

Spray tip penetration has been analyzed according to the comparison with available experimental data by Kim et al. [13]. Spray tip penetration is defined as maximum length achieved by the spray droplets during the injection period. The ambient pressure was kept constant at $0.1 \mathrm{MPa}$ for the test case. Spray tip penetrations btained from the experiment were measured either by Phase Doppler Anemometry (PDA) or photographically. For the calculations it is determined as the axial position behind which $90 \%$ of the liquid volume are contained. Included are most of the relative interactions between spray droplets and ambient gas as modelled in spray sub-models. Figure (2) shows the calculated spray tip penetration in comparison with experimental data. The results show the agreement between simulation and experimental data is very satisfactory. According to this figure, time-dependent development of 
the spray tip penetration length can be divided into two intervals. The first time interval begins at the beginning of injection $(\mathrm{t}=0 \mathrm{msec})$ when the needle starts to open and ends at the moment the liquid droplets emerging from the nozzle hole begin to disintegrate (here until $\mathrm{t}=1 \mathrm{msec}$ ). The simulated results are over predicted as compared with the experimental data because of the small needle lift and the low mass flow rate at the beginning of injection. This leads the injection velocity to be small. During the second interval $(\mathrm{t}>1 \mathrm{msec})$, the spray tip penetration simulated results presented the good consistent with the experimental results. The reason is the spray tip velocity is approximately close to the experimental velocity during the second period.

Now the aim of this study will start by comparing three mainly parameters to characterized Soybean Oil Methyl Ester (SME). Firstly, Figure (3) shows the liquid volume faction at time $2 \mathrm{msec}$. To figure out the distribution of the liquid volume fraction, the axial direction was selected to show the way in which the liquid droplets are spread out. From this contour plot the liquid volume or concentration increases near the injector due to the liquid mass has been injected for $1.2 \mathrm{msec}$. whereas it can be noticed that at the front of the spray as the less concentration is found because it is associated with the atomization processes. The liquid volume fraction in diesel exhibited more penetration and wide cone angle than the (SME). This is because, the most important reason described here in this work is the fuel physical properties. For example the fuel density can be affected on the atomization process and spray tip penetration by decelerating the injection delay time as will be presented below.

$$
U_{i n j}=c d \sqrt{\frac{2\left(P_{i n j}-P_{a m b}\right)}{\rho_{l i q}}}
$$

Figure (4) shows contour plot for a comparison between diesel and (SME) at time 2.0 msec. After the start of injection of liquid, the smaller droplets were seen to move towards the center of spray because of the entrained gas velocity induced by the spray. Also the Sauter mean diameter can be defined as $(\mathrm{SMD}=\mathrm{Q} 3 / \mathrm{Q} 2)$. That explain the highest values can be found at the axis of symmetry. 


\section{No.16 Journal of Petroleum Research \& Studies}

$(\mathbf{J P R \& S})$

In order to obtain a good agreement with the experimental data, the inlet boundary conditions were implemented according to the data provided by Kim et al [14]. To minimize the error of the pressure variation, the injection pressure variation with time was overcome using the injection pressure profile. In the first $0.2 \mathrm{~ms}$, the injection pressure increases linearly with time from its initial value of $40 \mathrm{MPa}$ to a maximum value of $50 \mathrm{MPa}$. As a consequence the computed injection velocity follows similar trends injection. The discharge coefficient was set at a fixed value of 0.7 .

Figure (5) compares the predicted results of the liquid velocity components for the diesel and (SME) liquid fuels after $2.0 \mathrm{~ms}$. The comparison used the third moment velocity components as they are closer in nature than area and drop radii-averaged velocities. The axial profile shows that the maximum velocity occurs at the core of the spray (at the axis_of symmetry). That is because large droplets which are heaviest (biggest momentum) are found here.

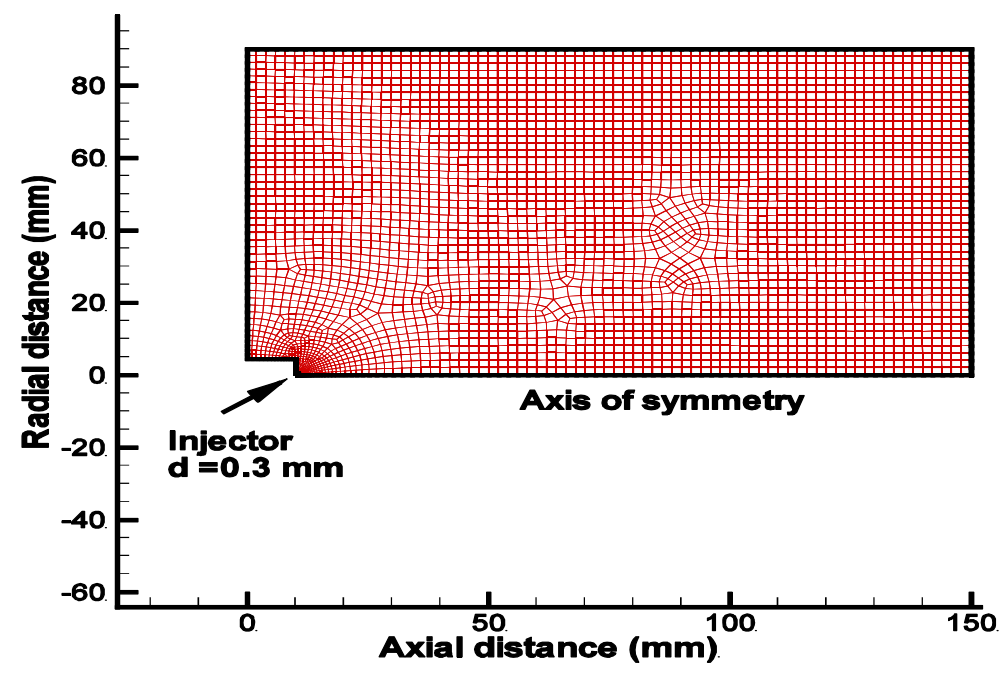

Fig. (1) Computational domain 


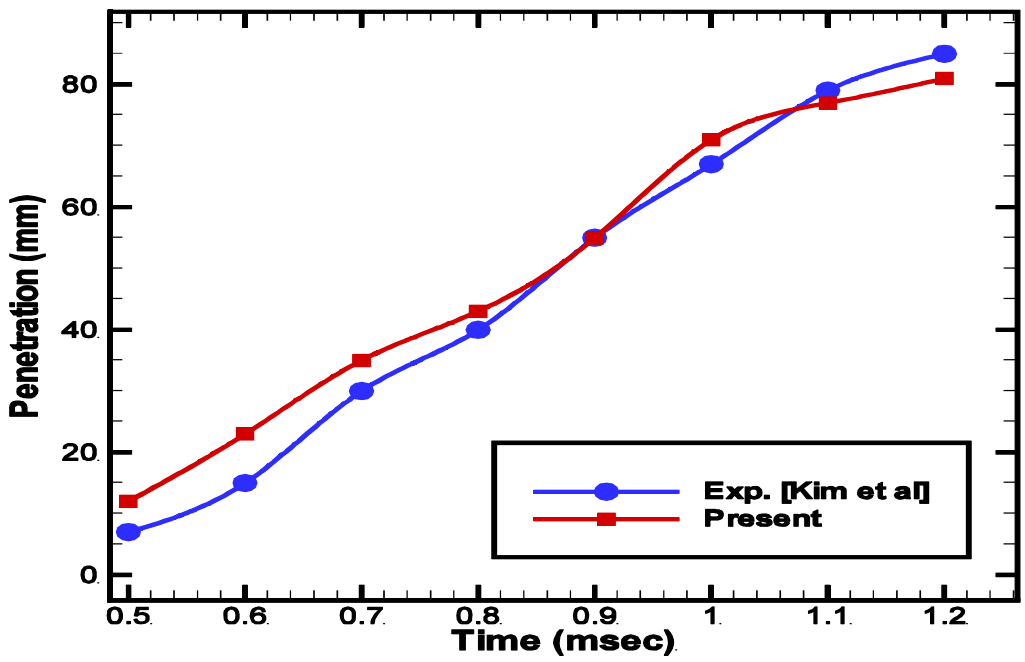

Fig. (2) Spray penetration

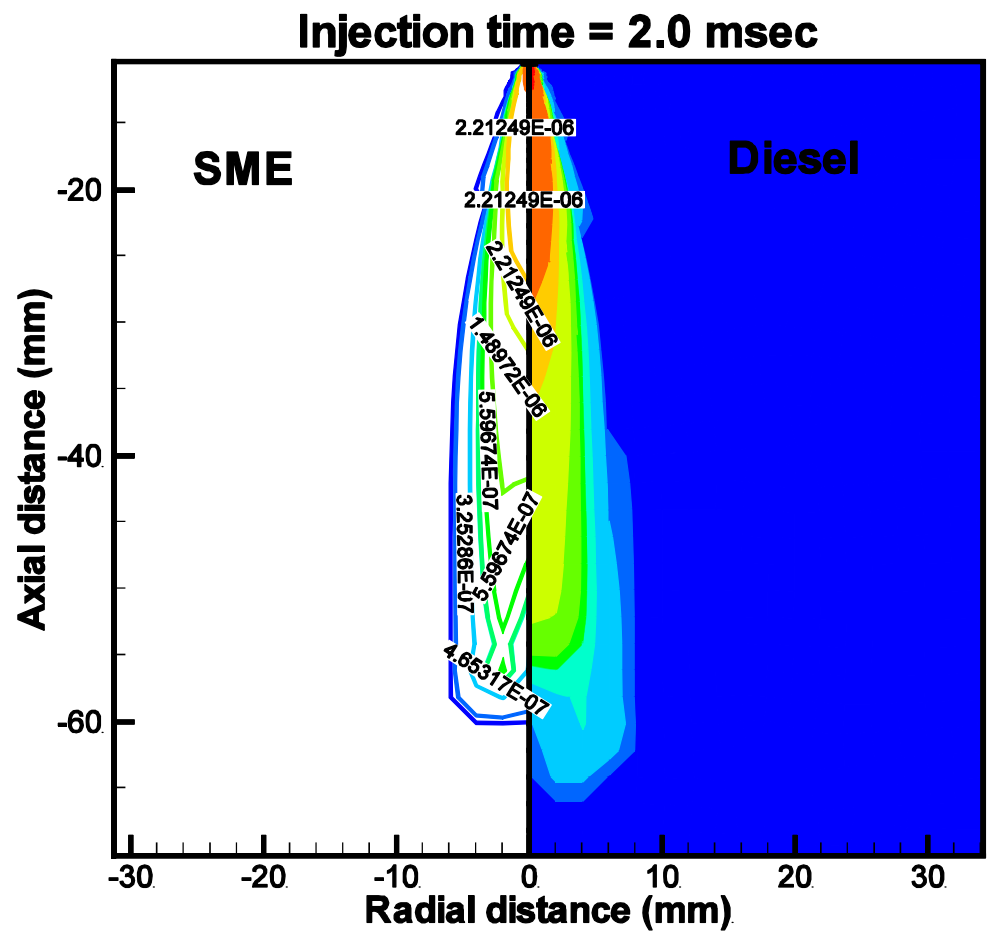

Fig. (3) Liquid volume fraction 


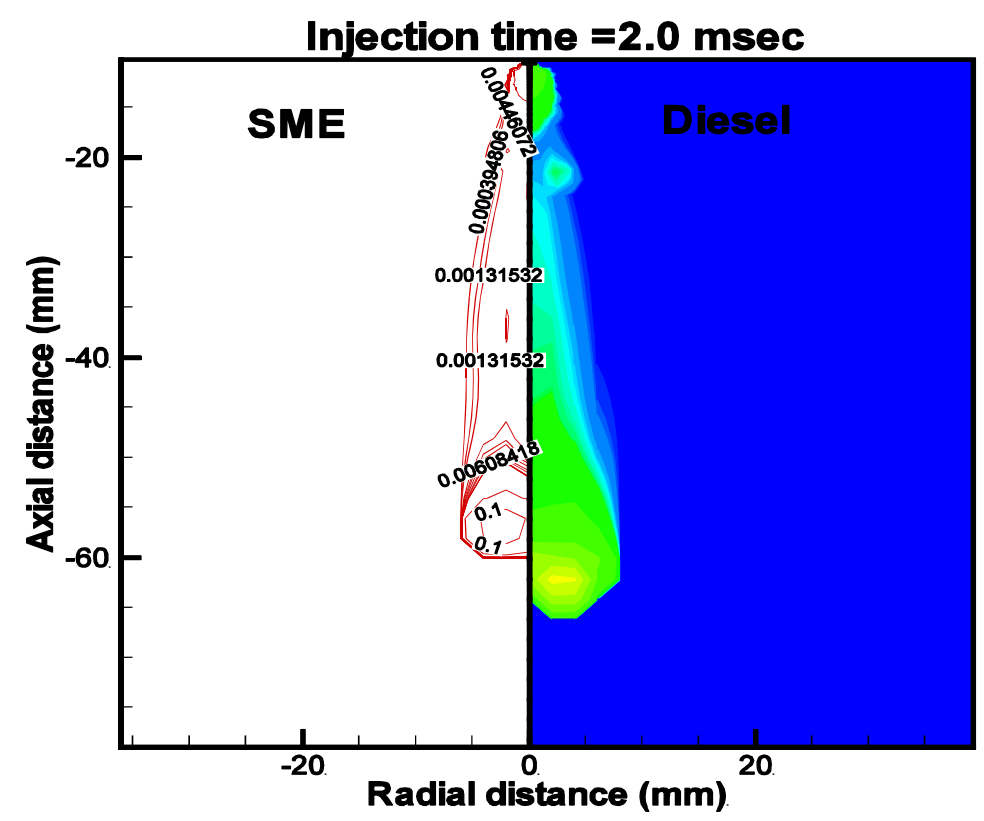

Fig. (4) Sauter mean diameter

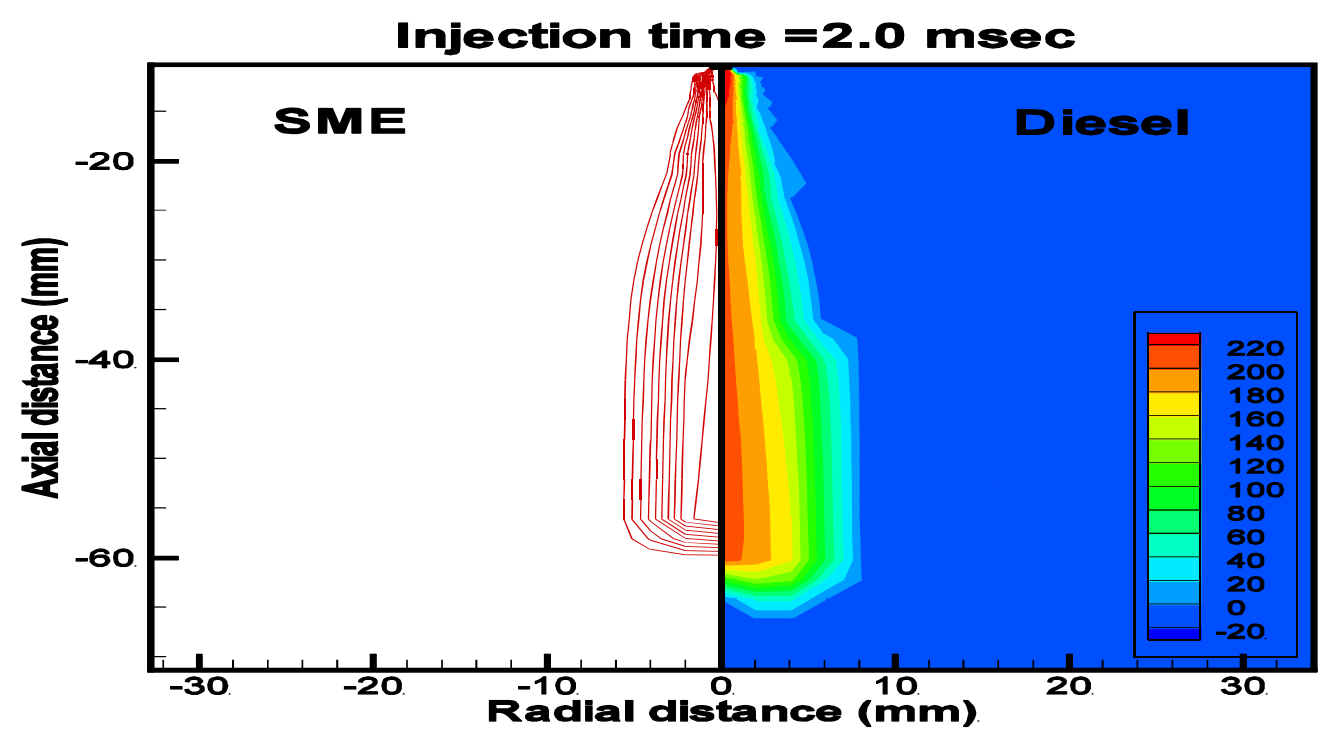

Fig. (5) Spray axial velocity 


\section{Conclusions}

The aim of this study is to perform an optimal numerical simulation for biodiesel spray in a common rail diesel engine. As is apparent from methods of computational, the advanced numerical techniques can perform well as compared with the experimental data. The conclusions that are obtained by this work are as follows:

1. The predicted results of the spray tip penetration presented the good consistent with the experimental results. The liquid volume fraction and surface area concepts (based on spray moment theory) can cope with interactions between the two-phases with less computational effort and more efficiently.

2. One of the main advantages to use the numerical simulation will be appeared here. The computational calculations can provide the comparison with the experiment by adding the range of droplet sizes. 


\section{References}

1. S. Lee, D. Tanaka, J. Kusaka, and Y. Daisho. Effects of diesel fuel characteristics on spray and combustion in a diesel engine; JSAE200224660, Japanese Society of Automotive Engineers: Tokyo, Japan, 2002.

2. C. S. Lee, S.W.Park, and S. I. Kwon. An Experimental Study on the Atomization and Combustion Characteristics of Biodiesel-Blended Fuels. Energy Fuels, 19 (2005), 2201.

3. Y. Wengiao. Computational modelling of nitrogen oxide Emissions from biodiesel based on accurate fuel properties. W. Yuan, PhD Dissertation, University of Illinois at Urbana Champaign. Department of Agricultural and Biological Engineering, 2006.

4. C. A. W. Allen. Prediction of biodiesel fuel atomization characteristics based on measured properties. PhD Dissertation, Dalhousie University DALTECH, Halifax, Canada, 1998.

5. K. Yamane, A. Ueta, and Y. Shimamoto. Influence of Physical and Chemical Properties of Biodiesel Fuel on Injection, Combustion and Exhaust Emission Characteristics in a DI-CI Engine, The Fifth International Symposium on Diagnostics and Modeling of Combustion in IC Engines COMODIA, Nagoya, Japan, July 14, 2001.

6. Su Han Park, Hyung Jun Kim, Hyun Kyu Suh and Chang Sik Lee. A study on the fuel injection and atomization characteristics of soybean oil methyl ester (SME). International Journal of Heat and Fluid Flow,30 (2009), 108-116.

7. Ahmed Abed Al-Kadhem Majhool and Abbas Alwi Sakhir ALJeebori. Study of modelling spray penetration of biodiesel fuel under transient engine conditions. Academic Research International. Vol. 3, No. 3, November 2012, 70-79.

8. J. C. Beck and A. P. Watkins. On the development of a spray model based on drop-size moments. Proc. R. Soc. Lond., A(459)(2003),1365-1394.

9. R.H. Windschitl, http://www.rskey.org/gamma.htm.

10. J. C. Beck and A. P. Watkins:On the Development of Spray Submodels Based on Droplet Size Moments. Journal of Computational Physics 182, 586621,2002.

11. F.H. Harlow, and A. A. Amsden: Numerical calculation of multiphase fluid flow. Journal of Computational Physics, 17, 19-52, 1975. 


\section{No.16 Journal of Petroleum Research \& Studies}

(JPR\&S)

12. J. C. Beck and A. P. Watkins. On the development of a spray model based on drop-size moments. Proc. R. Soc. Lond., A(459):13651394,2003a.

13. Hyung Jun Kim, Su Han Park and Chang Sik Lee. A study on the macroscopic spray behavior and atomization characteristics of biodiesel and dimethyl ether sprays under increased ambient pressure. Fuel Processing Technology 91 (2010) 354363

14. R. I. Issa. Solution of the implicitly discretised fluid flow equations by operator splitting. Journal of Computational Physics, 61, 40- 65, 1986. 